\title{
Finite Element Analysis of Vibrating Canisters Filled with Calcined Nuclear Waste
}

\section{Yun-Bo Yi*}

Department of Mechanical and Materials Engineering, University of Denver, USA

A finite element analysis is performed to investigate the dynamic responses of canisters during the vibrational compaction of calcined radioactive waste. This study shows that the maximum internal stress consistently occurs in the canister wall, but its actual location varies and depends on the loading and boundary conditions. The mechanical properties of the granulated calcined nuclear waste have appreciable impact on the internal stress of the canister container at higher fill percentage. Finally, a set of loading parameters has been suggested for safe operation of the canister system.

Calcine is a more desirable material to store than liquid radioactive waste because it reduces volume, is much less corrosive, less chemically reactive, less mobile under most conditions, easier to monitor and more protective of human health and the environment. This paper describes the technical issue involved in the development of a feasible solution for further volume reduction of calcined nuclear waste for transportation and long term storage. This will be accomplished by developing a process wherein the canister is transformed into a vibrational machine, for further volume reduction by about $35 \%$. The random compaction experiments show that this volume reduction is achievable. The main goal of this paper is to demonstrate through computer modeling that it is feasible to use volume reduction vibrational machine without developing stress/strain forces that will weaken the canister integrity.

In the study, a canister was simplified as a cylindrical container of uniform wall thickness. No damping factors were incorporated in the model. This significantly reduced the computational time without losing accuracy. However, this simplification was restricted to the vertical modes of oscillation only, and inapplicable to the horizontal shaking modes. In simulating the vertically modes, a vibrating harmonic displacement was applied along the axial direction at the bottom of the canister. The nonlinear dynamic analysis was performed by using the commercially available code ABAQUS/Explicit [1] through the explicit direct integration option. A full cycle of the sinusoidal load was simulated to capture the fundamental features of the problem. Stainless steel properties have been used for the canister container. The waste material is primarily composed of calcium fluoride. However, the effective elastic modulus and the density of particles are much different from those of continuous solid [2,3]. Here the modulus of $\mathrm{CaF}_{2}$ particles was assumed proportional to the mass density. Since the density of solid $\mathrm{CaF}_{2}$ is $3.18 \mathrm{~g} / \mathrm{cm}^{3}$ and the modulus is $75.8 \mathrm{GPa}$, and the measured density of the waste material is $1.3 \mathrm{~g} / \mathrm{cm}^{3}$, we estimated the effective modulus of the granular material to be $31.0 \mathrm{GPa}$.

The natural frequency response was the first thing examined for possible structural resonance. It turns out that for an empty canister, the first natural frequency is approximately $65 \mathrm{~Hz}$. When the canister is completely filled with the waste material, the frequency is around 146 $\mathrm{Hz}$. This range of the natural frequency is much higher than the applied loading frequency. Therefore our conclusion is that a horizontal shaking mode will not excite system resonance either. Further, a reexamination of the first natural frequency of the vertical mode for the empty canister shows a result of $382 \mathrm{~Hz}$, which is very close to the result obtained from the axisymmetric model. The small discrepancy could arise from the different mesh refinements used in the two models.

The lateral shaking mode shows that the maximum Von Mises stress increases with the load frequency, with only a slight variation. The increase rate or the slope of the curve is about $20 \mathrm{MPa} / \mathrm{Hz}$. This is much smaller than the vertical mode, which shows a rate of $50 \mathrm{MPa} /$ $\mathrm{Hz}$. Therefore, at the same load frequency, the vertical modes induce higher internal stress than the horizontal modes. The maximum stress also increases with fill percentage of the waste material. In comparison with the vertical mode, the waste material plays a more important role here: in an empty canister, the maximum stress is slightly above 50 $\mathrm{MPa}$, whereas at the $100 \%$ fill, the stress increases significantly to 300 $\mathrm{MPa}$. It has also been noticed from the curve that the increase in the stress mainly occurs when the fill percentage is greater than $50 \%$.

The yield stress for stainless steel varies, but typically below 300 $\mathrm{MPa}$. It has been noticed that some of the computed maximum stresses, especially for the vertical modes, are much higher than this threshold and therefore the canister is unsafely designed. Apparently the internal stress will decrease when the amplitude of vibration is attenuated. For example, when a standard canister filled with $80 \%$ of the calcined waste operates under a load frequency $10 \mathrm{~Hz}$ and amplitude $0.005 \mathrm{~m}$, the maximum stress in both vertical and horizontal vibration modes will be below the yield stress.

The following conclusions have been obtained from this study:

i. The maximum stress concentration always occurs inside the canister wall.

ii. The vertical vibration mode typically induces higher internal stress than the lateral mode.

iii. The first natural frequencies of the canister are generally much greater than the applied loading frequencies and therefore the structure resonance that may cause damage to the canister is unlikely to occur when the suggested parameters are used.

iv. The mechanical properties of the granulated calcined nuclear waste may have significant impacts on the internal stress of the canister container.

*Corresponding author: Yun-Bo Yi, Associate Professor, Department of Mechanical and Materials Engineering, University of Denver, USA, E-mail: Yun-Bo.Yi@du.edu

Received September 05, 2013; Accepted September 07, 2013; Published September 09, 2013

Citation: Yi YB (2013) Finite Element Analysis of Vibrating Canisters Filled with Calcined Nuclear Waste. J Appl Mech Eng 2: e117. doi:10.4172/21689873.1000e117

Copyright: (c) 2013 Yi YB. This is an open-access article distributed under the terms of the Creative Commons Attribution License, which permits unrestricted use, distribution, and reproduction in any medium, provided the original author and source are credited. 
Citation: Yi YB (2013) Finite Element Analysis of Vibrating Canisters Filled with Calcined Nuclear Waste. J Appl Mech Eng 2: e117. doi:10.4172/21689873.1000 e117

Page 2 of 2

Finally, the calculated data suggest that by appropriate selection of the loading parameters, we are able to maintain the maximum internal stress below a certain threshold so that the entire canister can operate safely without defects. This will have significant economical benefits of handling nuclear waste in the calcined forms, including waste manipulation, storage and transportation.

\section{References}

1. Simulia Inc (2009) ABAQUS/Explicit User's Manual, Version 6.9. Providence, RI, USA.

2. Knight JB, Fandrich CG, Lau CN, Jaeger HM, Nagel SR (1995) Density Relaxation in a Vibrated Granular Material. Phys Rev E51: 3957-3963.

3. Nowak ER, Knight JB, Ben-Naim E, Jaeger HM, Nagel SR (1998) Density Fluctuations in Vibrated Granular Materials. Phys Rev E57: 1971-1982. 